\title{
OPTIMAL PRICING FOR MOBILE VIDEO STREAMING USING BEHAVIOR ANALYSIS
}

\author{
W. Sabrina Lin and K. J. Ray Liu \\ ECE Dept., University of Maryland, College Park, MD 20742 USA
}

\begin{abstract}
Mobile phones are among the most popular consumer devices and the recent developments of $3 \mathrm{G}$ networks and smart phones enable users to watch video programs by subscribing data plans from service providers. Due to the ubiquity of mobile phones and phone-tophone communication technologies, subscribers can redistribute the video content to non-subscribers. Such a redistribution mechanism is a potential competitor for the service provider and is very difficult to trace given the users' high mobility. The service provider has to set a reasonable price for the data plan to prevent such re-distribution behavior to protect his/her own profit. In this paper, we analyze the optimal price setting for the service provider by investigating the equilibrium between the subscribers and the secondary buyers in the content-redistribution network. We model the behavior between the subscribers and the secondary buyers as a hybrid Stackelburg auction game and find the optimal price and quantity for both groups of users. Such an analysis can help the service provider preserve their profit under the threat of the redistribution networks and can improve the quality of service for end users.
\end{abstract}

\section{INTRODUCTION}

The explosive advance of multimedia processing technologies are creating dramatic shifts in the ways that video content can be consumed and delivered by end users. Also, the spread of wireless network accessibility and mobile devices is drawing lots of attentions on ubiquitous multimedia access within the multimedia community in the past decade. Network service providers and researchers are focusing on developing efficient solutions for the ubiquitous access to multimedia data and in particular videos, from everywhere with mobile devices (laptops, PDAs or the smart cellular phones that can access $3 \mathrm{G}$ networks)[1]. Mobile-phone users can watch video program over their devices by subscribing to the data plans from network service providers $[2,3]$. These end users can retrieve and reproduce the video content from the network thanks to the programmable hand devices. Therefore, it is important to understand the possible actions of the end users in order to provide better ubiquitous video access services.

According to the survey on mobile devices popularities[4], almost every person has at least one cellphone in developed countries. Combining such a high popularity and the phone-to-phone communication enabled by various technologies, it is very possible for dataplan subscribers to redistribute the video content. For example, some users who do not subscribe to the data plan may be willing to watch tv programs while waiting for or on public transportation; some of them might want to check most the updated news at anytime. Hence these users have incentives to buy the desired video from neighboring data-subscribers if the cost is lower than subscribing from the service provider. Unlike generic data, multimedia contents are easy to be retrieved and modified on the device, and such a property

The authors can be reached at wylin@umd.edu and kjrliu@umd.edu makes the redistribution of the video content even more possible. On the other hand, the subscribers also have incentives to redistribute the content with the price higher than their transmission cost as long as such an action will not be detected by the content owner. Due to high mobility, high time-sensitiveness, and small transmission range of the mobile devices, each redistribution action only exists for a short time. Thus, such a redistribution network is very difficult to track. Consequently, a better way to prevent copyright infringement is to set a price that no subscribers will have incentives to redistribute the video.

The content subscribers and the secondary buyers who are interested in the video data interact with each other and influence each others' decisions and performance. Both groups of users will reach agreement at the equilibrium price that all users have no incentive to deviate. Hence, such an equilibrium price will serve as the upper bound for the price set by the network service provider. Due to the small coverage and limited power of each mobile device, a subscriber can only sell the content to the secondary buyer within his/her transmission range, and the distance and channel conditions between users dominate the users' decisions. As a very first work on this problem, we focus on the case that there is only one secondary buyer that the subscribers can sell to. To solve this hybrid user dynamics in the live-video marketing social network, we propose a multiuser game [5] to solve the problem.

The rest of the paper is organized as follows. We introduce the system model and define the problem and the utility functions for the subscriber and the secondary buyer in Section 2 . We then analyze the optimal strategies for all users and provide the solutions in Section 3. Simulation results are shown in Section 4 and conclusions are drawn in Section 5.

\section{SYSTEM MODEL}

In this section, we will introduce the channel, transmission, and ratedistortion model for the video transmission.

The system diagram is shown in Figure 1. There are $N$ subscribers in the network, trying to sell the video stream to the secondary buyer. At the beginning, each subscriber send his/her own price per unit transmit power as well as the probing signal to the secondary buyer. Since the price information contains only a few bits, we assume that it can be received immediately and perfectly. The probing signal is meant to let the secondary buyer estimate the maximal achievable transmission rate. The secondary buyer has to decide how much power he/she wants to buy from each subscriber. Since scalable video coding is widely used in mobile video streaming [6], the secondary buyer can purchase different layers of the video stream from different subscribers and combine these streams during decoding processes.

Suppose the $i^{t h}$ subscriber $S_{i}$ is transmitting the video chunks to the secondary buyer $B$ using power $P_{i}$, the channel between them is slow fading channel with channel gain $H_{i j}$, the distance between them is $d_{i}$ and the variance of the additive white gaussian noise at 


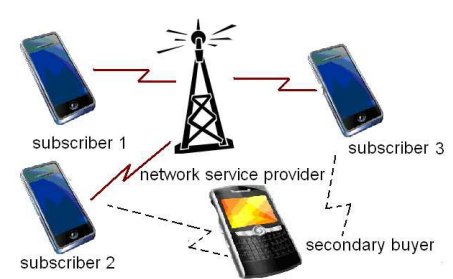

Fig. 1. An example of a mobile video-stream redistribution network

the receiver side is $\sigma^{2}$, then the signal-to-noise ratio (SNR) and the maximal achievable bit rate of the video stream is between $S_{i}$ and $B$ can be expressed by

$$
S N R_{i}=\frac{P_{i} H_{i}}{\sqrt{d_{i}} \sigma^{2}}, \text { and } R_{i}=W \log _{2}\left(1+\frac{S N R_{i}}{\gamma}\right),
$$

where $W$ is the bandwidth of the for transmission, and $\gamma$ is the capacity gap.

For a video streaming service, two common objective quality measure are the video's peak signal-to-noise-ratio (PSNR) and the streaming delay. Here we adopt the polynomial delay model as in [7]. The overall delay $D_{B}$ at the secondary buyer's end is the network delay between the subscribers and the service provider plus the maximal processing time of the subscribers. Therefore,

$$
D_{B}=D_{q}\left(\frac{N^{\prime}+K}{M}\right)+\max _{i \in N^{\prime}} D_{p}(i)
$$

where $N^{\prime}$ is the number of subscribers that the secondary buyer purchase the video stream from, $M$ is the maximal number of user that the network service provider can afford simultaneously, $D_{q}\left(N^{\prime}+\right.$ $K)$ is the network delay between the subscribers and the service provider, $K$ is the number of other subscribers within the coverage of the same base station but cannot establish direct link to the secondary buyer, and $D_{p}(i)$ is the processing time of subscriber $i$.

The PSNR of the video stream between $S_{i}$ and $B$ is $P S N R_{i}=$ $10 \log _{10} \frac{255^{2}}{M S E_{i}}$, where $M S E_{i}$ is the mean square error which is the distortion of the reconstructed video. Without loss of generality, in this paper, we use the two-parameter rate-distortion model, which is widely employed in a medium or high bit-rate situation, and other models can be similarly analyzed. Note that the secondary buyer is able to purchase the video from different subscribers in two different ways. It is easy to prove that since the log function and the exponential function are convex over $\mathbb{R}^{+}$and the exponential functions is non-decreasing over $\mathbb{R}^{+}$, buying different video-stream layers from different subscribers is a better choice. One is asking the subscribers to send the same bit stream, the other is asking the subscribers to send different layers of the video and combine bit streams in decoding process. Since the video bit rate is formulated in (1), the mean square error of the reconstructed video stream can be expressed by

$$
M S E=\alpha e^{-\beta \frac{W}{N^{\prime}+1} \sum_{i \in N} \log _{2}\left(1+\frac{S N R_{i}}{\gamma}\right)}
$$

where $\alpha$ and $\beta$ are two positive parameters determined by the characteristics of the video content.

\section{ANALYSIS OF OPTIMAL STRATEGIES}

In this section, we will model the behavior of the subscribers and the secondary buyer as a Stabkelburg game, and then analyze the equilibrium, which leads to the optimal strategies for all users.

\subsection{Video-Stream Redistribution Game}

Since the video-stream redistribution network is a dynamic system, in which all users have very high mobility and users can join and leave at anytime, it is very difficult to control the user behavior by a central authority. On the other hand, since redistributing infringes copyrights, the users (subscribers and secondary buyers) have incentives to not trust one extra person (the central authority) to minimize their risk of being detected by the service provider. Hence, we propose a fully-distributed Stackelburg-game-theoretical model to analyze how secondary buyer provide incentives for the subscribers to redistribute the video stream, and what is the optimal price and quantity that the secondary buyers should offer. The ultimate goal of such analysis is to help the content owner, i.e. the service provider to set the price such that the equilibrium of the game between subscribers and the secondary buyers leads to negative payoff, which means the subscribers have no incentive to redistribute the video. We start the analysis by the defining the stages of the game and the utility functions of both types of users in the network.

- Game Stages: Before the game starts, each user, either a subscriber or the secondary buyer, will declare his/her presence to let all users within his/her transmission range.

The first stage of the game is the subscribers' (leaders') move. For each subscriber $i$, he/she will set the unit price $p_{i}$ per his/her transmission power as well as the maximal power $P_{i}^{(\max )}$ that he/she can use for transmission. Let $L$ be the set of all subscribers.

Then in the second stage of the game, the secondary buyer (follower) will decide whom to buy the video from and how much power he/she wants the subscriber to transmit. The secondary buyer will offer each subscriber the quantity of transmission power $P_{i}$.

- Utility Function of the secondary buyer/follower: We first discuss the utility function and the optimal action for the secondary buyer. The secondary buyer $B$ gain the reward by successfully receiving the video with a certain quality. On the other hand, $B_{i}$ has to pay for the power that the subscribers use for transmission. Therefore, given the video rate-distortion model, the utility function of a secondary buyer $B_{i}$ can be defined as

$$
\begin{aligned}
\pi_{B}=g_{Q}(P S N & \left.R_{B}-P S N R_{\max }\right)-g_{D}\left(D_{B}-D_{q}\left(\frac{K+1}{M}\right)\right) \\
& -\left(\sum_{i \in N^{\prime}} p_{i} P_{i}+p_{o}\right)
\end{aligned}
$$

where $g_{Q}$ and $g_{D}$ are the user-define reward terms for the two streaming quality measure, the visual quality and the streaming delay, respectively. $P S N R_{\max }$ is the maximal PSNR of the video which can be obtained by buying the stream service from the content owner, $p_{o}$ is the price set by the content owner.

(4) can be viewed as the difference between the utility that the secondary buyer obtains from buying the stream service from the subscribers from subscribing the video stream from the service provider directly. The first term in (4) reflects the visual quality difference between the subscriber's video stream and the service provider's video stream. The second term is the delay difference between the subscriber's video stream and the service provider's video stream. $D_{B}$ was defined in (2), and $D_{q}(K+1)$ is the delay profile if the secondary buyer subscribes the data plan and acts as an extra subscriber in the network. And the third term indicates the difference between the payments. $g_{Q}$ and $g_{D}$ control the balance between the gain and cost for the secondary buyer.

- Actions of subscribers/auctioneers/seller:

Each subscriber $S_{i}$ can be viewed as a seller and aims to not only earn the payment that covers his/her transmission cost but also gain 
as much extra reward as possible. We introduce a parameter $c_{i}$, the cost of power for relaying data, in our formulation. $c_{i}$ is determined by the characteristics of the device that subscriber $S_{i}$ uses. Hence, the utility of $S_{i}$ can be defined as $\pi_{S_{i}}=\max _{j}\left(p_{i}-c_{i}\right) P_{i}$, where $P_{i}$ is the power that subscriber $i$ used for transmission.

The choice of the optimal price $p_{i}$ is affected not only by each subscriber's own channel conditions to each secondary buyer but also by the other subscribers' prices. This is because the sellerlevel game is noncooperative, and the relay nodes compete to get selected by source node $s$. If a certain subscriber $S_{i}$ asks such a high price that makes it less beneficial than the other subscriber to the secondary buyer, then secondary buyer will buy less from subscriber $S_{j}$ or even discard it. It is worth noticing that the only signaling required to exchange between the source node and the relay nodes are the price $p_{i}$ and the information about how much power $P_{i}$ to buy. Consequently, the proposed two-level game-theoretical approach can be implemented in a distributed way. The outcome of the proposed games will be shown in detail in the following section.

\subsection{Equilibrium Analysis}

The video-stream redistribution game is with perfect information: the game is composed of two stages, the subscribers make decisions first, followed by the secondary buyer. Since each subscriber's action is setting $p_{i}$, and he/she has to enclose the price to the secondary buyer, the secondary buyer knows each subscriber's strategy. As a result, each information set in this game contains only one node, which shows the video-stream redistribution game is with perfect information.

According to backward induction [5], a game with perfect information has at least one equilibrium. Therefore the optimal strategies for both the secondary buyer and subscribers exist and can be obtained by solving the optimal decision for each stage in the backward manner.

\subsubsection{The secondary buyer' best strategy}

We analyze the game in the backward manner by investigating the optimal strategy for the secondary buyer first. The goal of the nonsubscriber $B$ is to determine the optimal power $P_{i}$ that $B$ should buy from each subscriber in $L$ to maximize his/her own utility as defined in (4).

Let $R_{B}$ be the video rate that the secondary buyer get from the subscribers,

$$
R_{B}=\left\{\frac{W}{\sum_{i \in L} \mathbb{1}\left(P_{i}\right)+1} \sum_{i \in L} \log _{2}\left(1+\frac{P_{i} H_{i}}{\gamma \sqrt{d_{i}} \sigma^{2}}\right)\right\},
$$

then according to the rate-distortion model in (3) and the transmission rate given in (1), the first term in (4) can be formulated as a function of the transmission rate as

$$
g_{Q}\left(P S N R_{B}-P S N R_{\max }\right)=g_{Q}^{\prime}\left(R_{B}-R_{\max }\right),
$$

where $g_{Q}^{\prime}=10 g_{Q} \beta / \log _{10}$ and $R_{\max }$ is the video rate provided by the content owner.

Combining (1) and (4) with the above equation, we can formulate the utility function of $B$ as a function of $\left\{P_{i} \forall i \in L\right\}$. According to [7], the network delay of the $3 \mathrm{G}$ network is reciprocal to the network utilization percentage. Hence the optimal strategy for the secondary buyer is

$$
\max _{P_{i}} g_{Q}^{\prime}\left(R_{B}-R_{\max }\right)-g_{D}\left[\max _{i \in L} D_{p}(i) \mathbb{1}\left(P_{i}\right)+D_{B}\right]
$$

$$
\begin{gathered}
-\left(\sum_{i \in L} p_{i} P_{i}-p_{o}\right) \\
\text { s.t. } R_{B} \leq R_{\max }, P_{i} \leq P_{i}^{(\max )} \forall i \in L, \text { where } \\
D_{B}=\frac{M C}{M-K-\sum_{i \in L} \mathbb{1}\left(P_{i}\right)}-\frac{M C}{M-K-1},
\end{gathered}
$$

and $C$ is the network constant.

Note that in (7) and (5), $\sum_{i \in L} \mathbb{1}\left(P_{i}\right)$ is a piecewise continuous function, and so as $\max _{i \in L} D_{p}(i) \mathbb{1}\left(P_{i}\right)$. Therefore the optimization cannot be solved at once for the whole feasible set and has to be divided into subsets. Without loss of generality, suppose $D_{p}(1) \geq$ $D_{p}(2) \geq \ldots \geq D_{p}(N)$, define subset $\mathbb{S}_{N^{\prime}}^{(k)}=\left\{\mathbf{P} \mid \sum_{i \leq N} \mathbb{1}\left(P_{i}\right)=\right.$ $\left.N^{\prime}, \sum_{i \leq k-1} P_{i}=0\right\}$, where $\mathbf{P}=\left(P_{1}, P_{2}, \ldots, P_{N}\right)$.

It is easy to prove that (7) is a convex optimization problem over the feasible subset $\mathbb{S}_{N^{\prime}}^{(k)}$. Hence, we can solve the optimal power vector $\mathbf{P}_{N^{\prime}}^{(k)}$ for subset $\mathbb{S}_{N^{\prime}}^{(k)}$ by taking the derivatives of $\pi_{B}$ with respect to $P_{i}$ :

$$
\frac{\partial \pi_{B}}{\partial P_{i}}=g_{Q}^{\prime} \frac{W \ln 2}{N^{\prime}+1} \frac{A_{i}}{1+A_{i} P_{i}}-p_{i}=0 \forall S_{i} \in L,
$$

where $A_{i}=\sqrt{d_{i}} \sigma^{2} \gamma / H_{i}$. Therefore, if the secondary buyer purchases from any $N^{\prime}$ subscribers with the same maximal processing delay,

$$
P_{i}\left(\mathbb{S}_{N^{\prime}}^{(k)}\right)=\frac{g_{Q}^{\prime} W \ln 2}{p_{i}\left(N^{\prime}+1\right)}-\frac{1}{A_{i}} \quad \forall S_{i} \in L
$$

is the maximizer. Note that (9) can be proved to be the unique maximizer for the feasible set $\mathbb{S}_{N^{\prime}}^{(k)}$ by finding the maximizer on the boundary. According to (9), given the same maximal processing delay and the same number of subscribers, the higher the price $p_{i}$, the less the power that the secondary buyer is going to purchase. Also, the secondary buyer tends to purchase more power from the subscribers that the signal attenuations in between are less.

After the maximizer over each feasible subset is obtained, the secondary buyer should choose the one which gives himself/herself the largest utility. Let $\mathbf{P}_{i}^{*}$ be the optimal decision of the secondary user, then $\mathbf{P}_{i}^{*}=\max _{0 \leq N^{\prime}, k \leq N} \pi_{B}\left(\mathbf{P}\left(\mathbb{S}_{N^{\prime}}^{(k)}\right)\right)$

\subsubsection{Subscribers' best strategies}

Given the optimal strategy $\mathbf{P}^{*}$ of the secondary buyer derived above, each subscriber $S_{i} \in L \backslash L_{c}$ seek to maximize their utility by setting the optimal price $p_{i}$ that

$$
\max _{\left\{p_{i}\right\}} \pi_{S_{i}}=\left(p_{i}-c_{i}\right) P_{i}^{*} .
$$

The optimal price $p_{i}^{*}\left(\mathbf{H}_{i}, \mathbf{d}_{i}\right)$ should satisfy

$$
\frac{\partial \pi_{S_{i}}}{\partial p_{i}}=P_{i}^{*}+\left(p_{i}-c_{i}\right) \frac{\partial P_{i}^{*}}{\partial p_{i}} \text { s.t. } p_{i} \leq c_{i} \quad \forall i \in L,
$$

or be on the boundary, which means $p *_{i}=c_{i}$. Note that the subscriber is willing to redistribute the video stream only if he/she can profit from the redistribution action. Therefore, they must not claim prince lower than their cost.

\section{SIMULATION RESULTS}

In this section, we will show the equilibrium of the video-stream redistribution game under different scenarios as well as the optimal price for the content owner. 
We set the coordinates of the secondary buyer as $(0 \mathrm{~m}, 0 \mathrm{~m})$, and the subscribers are uniformly located within the range of [50 m, -50 $\mathrm{m}]$ in both $\mathrm{x}$-axis and y-axis. The maximal transmit power $P_{\max }$ is $100 \mathrm{~mW}$, the noise level is $10^{-8} \mathrm{~W}$, and we select the capacity gap $\gamma=1$, bandwidth $\mathrm{W}=1 \mathrm{MHz}$, the gain weighting factors $g_{Q}=0.1$, $g_{D}=0.1 / \mathrm{ms}$, and the cost per unit of power for each subscriber $c_{i}$ is a realization of an uniform random variable within $[0.05,0.15]$. The processing delay of each subscriber, $D_{p}(i)$, is a realization of an uniform random variable within $[0.1,10.1] \mathrm{ms}$. We use the video sequence "Akiyo" in QCIF format and H.264 vodeio codec. The resulted rate-distortion parameter $\beta=0.0416$, and $\alpha=6.8449$. We set the maximal PSNR which is provided by the original content owner be $35 \mathrm{~dB}$, and the corresponding maximal bit-rate for Akiyo is $84 \mathrm{kB} / \mathrm{sec}$. The subscription price $p_{o}$ for the video sequence is set to be 0 so that the optimal price for the content owner can be simply view as $\pi_{B}$.

First let $M-K=50$, and the number of subscribers varies from 1 to 5 , which means the network is not crowded and the number of subscribers compared to the maximal number that the network can afford is less. In Fig. 2, we can observe that as the total number of the available subscribers increases, the competitions among the subscribers become more severe, so the optimal price for the content owner decreases. When there are no more than 3 subscribers, the averaged utility of the subscribers does not vary much since in these cases the secondary buyer is trying to purchase maximal video rates from all subscribers to increase the PSNR of the video, so these subscribers are not competing with each other. However, when there are more and more subscribers, the secondary buyer can easily get the video quality close to $P S N R_{\max }$, each subscriber is competing with other subscribers to motivate the secondary buyer to purchase from himself/herself. Such a phenomenon is the nature of free market with more sellers.

Next we will examine the impact of network quality on the optimal price of the video stream. From Fig 2 we can see that the competition among subscribers dominate their own utilities and the optimal price for the video stream does not vary much when there are more than 3 subscribers. Therefore, here we set the total number of subscribers be 3, and Fig. 3 shows how different video stream PSNR $R_{\max }$ offered by the content owner and network usage influences the optimal video stream price. In Fig. 2, $M-K$ varies from 5 to 50 , and $R_{\max }=30,35,40$, respectively. From Fig. 2, we can see that if the service provider can offer a good-enough video quality that is almost irreplaceable by the redistribution network, he/she can charge more for the streaming service. Also, when the network is very busy, the video delay dominates the video quality, therefore the secondary buyer has more incentives to purchase from less number of subscribers, but each subscriber can only provide limited video quality. Hence for the content owner, providing better-quality video stream advantages the content owner more when the network is busy. Furthermore, given the same $R_{\max }$, when the network delay is less enough, the content price starts to degrade since the redistribution network is enabled. However, when the network delay is so less that is negligible, the content price starts to get slightly higher since buying from the subscribers introduces more delay which comes from the device.

\section{CONCLUSION}

In this paper, we propose a game-theoretical approach for the optimal price setting over mobile video streaming networks. We aim to investigate the optimal price of the mobile video streaming service by analyzing the equilibrium price of the video stream redistributed by the subscribers. Consequently, the results provide a guideline for

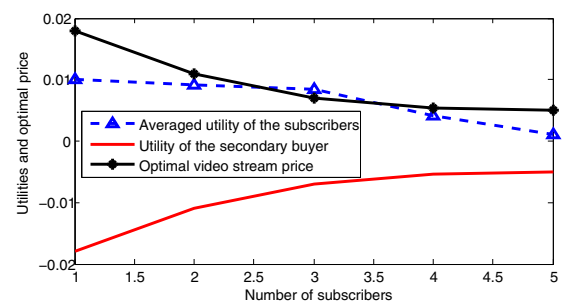

Fig. 2. Utilities of the users and the optimal video stream price versus different number of subscribers

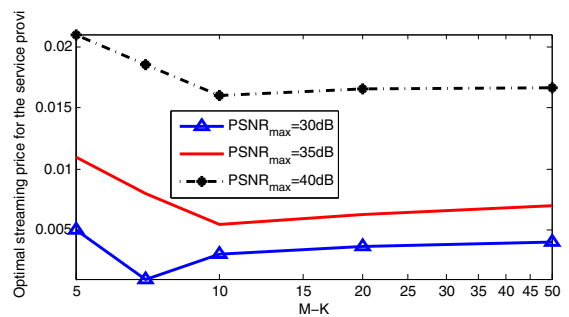

Fig. 3. Optimal video stream price versus qualities of network and streaming service

the content owner to prevent the redistribution behavior. We model the redistribution behavior as a Stackelburg game and analyze the optimal strategies of both subscribers and the secondary buyer who is willing to purchase the redistributed video stream. From the simulation results, the secondary buyer will tend to buy more power from the subscribers with better channel to maximize his/her utility, and if the total number of the subscriber increases, the secondary buyer can obtain a larger utility value, and the payment to each subscriber shrinks, due to more severe competitions among the sellers. Also, when the mobile phone network is crowded, the secondary buyer tends to purchase the video stream from less subscribers, and the price for the streaming service can be higher. Nevertheless, the service provider should always offer high-quality video stream to avoid competition with such a redistribution network.

\section{REFERENCES}

[1] G. Gualdi, A. Prati, and R. Cucchiara, "Video streaming for mobile video surveillance," IEEE Transactions on Multimedia, vol. 10, no. 6, pp. 1142-1154, Oct. 2008.

[2] D.F.S. Santos and A. Perkusich, "Granola: A location and bandwidth aware protocol for mobile video on-demand systems," in International Conference on Software, Telecommunications and Computer Networks, (SoftCom) 2008, Sept. 2008, pp. 309-313.

[3] S. Sudin, A. Tretiakov, R.H.R.M. Ali, and M.E. Rusli, "Attacks on mobile networks: An overview of new security challenge," in International Conference on Electronic Design, 2008., Dec. 2008, pp. 1-6.

[4] International Telecommunication Union, “"'http://www.itu.int/itud/ict/statistics/ict/graphs/mobile.jpg"," .

[5] G. Owen, Game Theory, Academic Press, 3rd edition, 2007.

[6] Heejung Lee, Yonghee Lee, Jonghun Lee, Dongeun Lee, and Heonshik Shin, "Design of a mobile video streaming system using adaptive spatial resolution control," IEEE Transactions on Consumer Electronics, vol. 55, no. 3, pp. 1682-1689, August 2009.

[7] G. Aniba and S. Aissa, "A general traffic and queueing delay model for 3G wireless packet networks," Lecture notes in computer science, $\mathrm{pp}$. 942-949, 2004. 